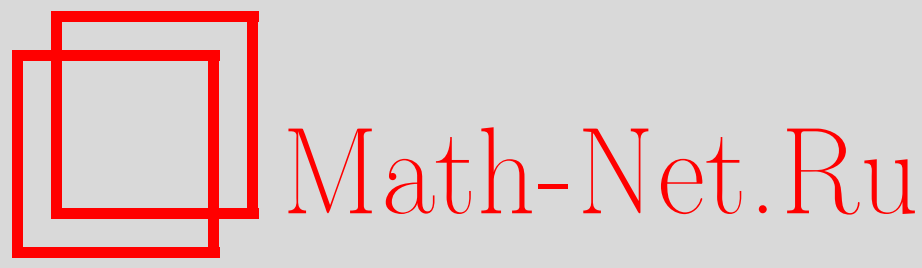

И. О. Глебов, В. В. Еремин, Новая форма уравнения Редфилда для диссипативных систем, связанная с матрицей корреляционных функций, ТМФ, 2009, том 161, номер 1, 83-94

DOI: https://doi.org/10.4213/tmf6421

Использование Общероссийского математического портала Math-Net.Ru подразумевает, что вы прочитали и согласны с пользовательским соглашением http://www.mathnet.ru/rus/agreement

Параметры загрузки:

IP : 3.85 .73 .92

26 апреля 2023 г., $17: 23: 31$

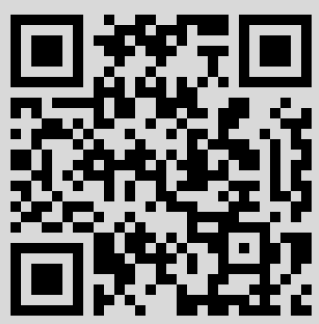




\title{
ФИЗИКА
}

Том 161, № 1

октябрь, 2009

2009 г.

И. О. Глебов*, В. В. Еремин*

\section{НОВАЯ ФОРМА УРАВНЕНИЯ РЕДФИЛДА ДЛЯ ДИССИПАТИВНЫХ СИСТЕМ, СВЯЗАННАЯ С МАТРИЦЕЙ КОРРЕЛЯЦИОННЫХ ФУНКЦИЙ}

\begin{abstract}
В рамках теории Редфилда рассмотрена задача о динамике колебательного движения в диссипативных системах. Гамильтониан взаимодействия наблюдаемой системы с термостатом разбивается на слагаемые, являющиеся произведениями оператора переходов системы и операторов перехода термостата. На основании этого разбиения построена матрица корреляционных функций, содержащая всю информацию о взаимодействии системы и термостата, и получена новая, операторная, форма уравнения Редфилда. Рассмотрены процедуры факторизации оператора взаимодействия и построения матрицы корреляционных функций. На основании диагонализации полученной матрицы заданы кореллированные операторы диссипации, введение которых упрощает операторную форму уравнений Редфилда. Показано, что для ряда задач, в которых возможно выбрать основные частоты переходов, данная процедура значительно сокращает размерность задачи о диссипативной динамике.
\end{abstract}

Ключевые слова: квантовая диссипация, теория Редфилда, матрица плотности, матрица корреляционных функций, оператор перехода.

\section{1. ВВЕДЕНИЕ}

В настоящее время учет релаксационных процессов становится одним из неотъемлемых элементов моделирования фемто- и пикосекундной молекулярной динамики. В первую очередь это связано со смещением интересов исследователей в сторону описания химических реакций и процессов переноса энергии, протекающих в системах с большим числом степеней свободы - биологических объектах (реакционных центрах и светособирающих антеннах фотосинтетических комплексов, нуклеиновых кислотах и т.п.) и других макромолекулярных системах [1]-[5].

Так, при описании динамики модельных систем, диссипативная динамика наблюдается уже при учете более чем трех степеней свободы [6]. Поэтому для молекулярных систем, состоящих из большого числа атомов, наиболее логичным кажется

${ }^{*}$ Московский государственный университет им. М. В. Ломоносова, Москва, Россия. E-mail: glebov_io@mail.ru,vv_er@mail.ru 
применение подходов, основанных на трактовке большей части системы как теплового резервуара и описании диссипативной динамики активных степеней свободы оставшейся части системы. Наиболее распространенный подход - теория Редфилда - использует формализм редуцированной матрицы плотности [7], [8].

Однако, несмотря на отсутствие необходимости явно учитывать все колебательные моды молекулы, задача о диссипативной динамике остается намного более емкой с вычислительной точки зрения, чем задача о когерентном движении такой же размерности в отсутствие диссипации. Это связано с тем, что при поэлементном численном решении уравнения Редфилда для систем с нерегулярными спектрами необходимо учитывать переходы между каждыми двумя электронно-колебательными состояниями. Тем не менее процедура разделения гамильтониана общей системы на термостат и динамическую часть во многом произвольна, что позволяет описывать динамическую часть и ее взаимодействие с термостатом с помощью точно решаемых квантовых моделей, например гармонического осциллятора. В настоящей статье предложен операторный подход к решению задачи о диссипативной динамике, основанный на выделении операторов, которые отвечают основным частотам перехода в системе, и позволяющий существенно снизить размерность тензора Редфилда. Этот подход применим для задач, в которых можно использовать приближения, позволяющие ограничить число учитываемых частот перехода на уровне динамической системы (гармоническое приближение и т.п.) или ее взаимодействия с термостатом.

\section{2. УРАВНЕНИЕ РЕДФИЛДА}

Для описания динамики, имеющей первоначально когерентный характер, а со временем приходящей к равновесному распределению, применимы методы, которые основаны на разделении объекта на две части: наблюдаемую (систему) и термостат. Полный гамильтониан с учетом разбиения на две части выглядит следующим образом:

$$
\mathbf{H}=H_{\mathrm{S}}+H_{\mathrm{B}}+V,
$$

где $H_{\mathrm{S}}$ - гамильтониан системы, учитывающий специфику процесса: число и структуру состояний, а также взаимодействие между ними, $H_{\mathrm{B}}$ - гамильтониан термостата, $V$ - гамильтониан взаимодействия между системой и термостатом.

Одной из наиболее распространенных теорий в рамках подхода "система + термостат" является теория Редфилда [7], основанная на следующих допущениях.

1. Влияние системы на термостат пренебрежимо мало, поэтому матрица плотности последнего соответствует равновесному тепловому распределению:

$$
\rho(t)=\rho_{\mathrm{S}}(t) \rho_{\mathrm{B}}(T), \quad \rho_{\mathrm{B}}(T)=\frac{e^{-\beta H_{\mathrm{B}}}}{Z},
$$

где $\rho_{\mathrm{S}}=\operatorname{tr}_{\mathrm{B}} \rho$ и $\rho_{\mathrm{B}}-$ матрицы плотности системы и термостата (матрица $\rho_{\mathrm{S}}$ называется редуцированной матрицей плотности (РМП), далее для простоты мы будем 
обозначать ее как $\rho) ; \beta=1 / k T$ - обратная температура; $Z$ - сумма по состояниям термостата; $\operatorname{tr}_{\mathrm{B}}$ обозначает след матрицы в базисе собственных состояний термостата.

2. Справедливо марковское приближение, согласно которому взаимодействие с термостатом уничтожает информацию о прошлых состояниях системы, поэтому временна́я производная РМП зависит только от текущего значения этой функции [8].

Уравнение движения для РМП в представлении взаимодействия выглядит следующим образом [8]:

$$
\frac{d}{d t} \rho_{\mathrm{SI}}(t)=-\frac{1}{\hbar} \operatorname{tr}_{\mathrm{B}}\left[V_{\mathrm{I}}(t), \rho_{\mathrm{SI}}(t) \rho_{\mathrm{B}}(T)\right]-\frac{1}{\hbar^{2}} \int_{0}^{\infty} \operatorname{tr}_{\mathrm{B}}\left[V_{\mathrm{I}}(t),\left[V_{\mathrm{I}}(t+\tau), \rho_{\mathrm{SI}}(t) \rho_{\mathrm{B}}(T)\right]\right] d \tau
$$

здесь и далее $T$ - температура, а индекс I или зависимость от времени для стационарных операторов обозначают представление взаимодействия: $V_{\mathrm{I}}(t)=e^{i\left(H_{\mathrm{S}}+H_{\mathrm{B}}\right) t / \hbar} \times$ $V e^{-i\left(H_{\mathrm{S}}+H_{\mathrm{B}}\right) t / \hbar}$.

Таким образом, для ряда задач первый член в (3) можно обратить в нуль, если надлежащим образом определить гамильтонианы термостата $H_{\mathrm{B}}$ и взаимодействия системы и термостата $V$ [8], поэтому далее мы будем использовать уравнение

$$
\frac{d}{d t} \rho_{\mathrm{SI}}(t)=-\frac{1}{\hbar^{2}} \operatorname{tr}_{\mathrm{B}} \int_{0}^{\infty}\left[V_{\mathrm{I}}(t),\left[V_{\mathrm{I}}(t+\tau), \rho_{\mathrm{SI}}(t) \rho_{\mathrm{B}}(T)\right]\right] d \tau
$$

Именно для этого базового уравнения мы выведем новую форму, использующую матрицу корреляционных функций.

\section{3. ФАКТОРИЗАЦИЯ ОПЕРАТОРА ВЗАИМОДЕЙСТВИЯ}

Во всех практически важных случаях оператор взаимодействия может быть представлен в виде [9]

$$
V=\sum_{j} F_{j} Q_{j}=\sum_{j>0} F_{j} Q_{j}+\sum_{j>0} F_{j}^{+} Q_{j}^{+}
$$

где операторы $F_{j}$ зависят только от координат системы, а операторы $Q_{j}$ - от координат термостата (для удобства примем обозначения $F_{-j}=F_{j}^{+}$и $Q_{-j}=Q_{j}^{+}$, подчеркнув этим эрмитовость оператора $V$ ). В этом случае оператор $V$ в представлении взаимодействия будет выглядеть следующим образом:

$$
\begin{gathered}
V_{\mathrm{I}}(t)=\sum_{j} F_{j}(t) Q_{j}(t), \\
F_{j}(t)=e^{i H_{\mathrm{S}} t / \hbar} F_{j} e^{-i H_{\mathrm{S}} t / \hbar}, \quad Q_{j}(t)=e^{i H_{\mathrm{B}} t / \hbar} Q_{j} e^{-i H_{\mathrm{B}} t / \hbar} .
\end{gathered}
$$


Разложение (5) оператора взаимодействия $V$ не является однозначным, так как любое линейное преобразование операторов $Q_{j}$ при соответствующем преобразовании операторов $F_{j}$ не меняет форму разложения:

$$
V=\sum_{j} F_{j} Q_{j}=\sum_{j} F_{j} \sum_{k} C_{j k} Q_{k}^{\prime}=\sum_{k} Q_{k}^{\prime} \sum_{j} C_{j k} F_{j}=\sum_{k} Q_{k}^{\prime} F_{k}^{\prime} .
$$

По этой причине мы можем сделать набор операторов $Q_{j}$ ортонормированным. Скалярное произведение определим через матрицу плотности термостата: $\left\{Q_{j}, Q_{k}\right\}=$ $\operatorname{tr}_{\mathrm{B}}\left(Q_{j}^{+} \rho_{\mathrm{B}}(T) Q_{k}\right)$.

При построении ортогонального набора будем отталкиваться от набора операторов $q\left(\omega_{j}\right)$ ( $\omega_{j}$ - частота перехода между двумя состояниями термостата), для которых выполняются условия

$$
\left[q\left(\omega_{j}\right), H_{\mathrm{B}}\right]=\omega_{j} q\left(\omega_{j}\right), \quad q\left(-\omega_{j}\right)=q^{+}\left(\omega_{j}\right)
$$

Набор этих операторов ортогонален, так как оператор $q^{+}\left(\omega_{j}\right) q\left(\omega_{k}\right)$ не содержит ненулевых диагональных элементов при $\omega_{j} \neq \omega_{k}$. Следовательно, операторы

$$
Q_{j}=\frac{q\left(\omega_{j}\right)}{\sqrt{\operatorname{tr}_{\mathrm{B}}\left(q^{+}\left(\omega_{j}\right) \rho_{\mathrm{B}}(T) q\left(\omega_{j}\right)\right)}}
$$

будут образовывать ортонормированную систему.

Отметим, что на динамику системы влияют только те частоты термостата, которые совпадают с частотами переходов в системе (это утверждение будет доказано в разделе 4). Благодаря этому в разложении (5) можно ограничиться только теми операторами $Q_{j}$, которые соответствуют переходам между собственными состояниями системы. Разложим произвольный оператор взаимодействия по ортонормированной системе $Q_{j}$ :

$$
V=\sum_{j} f_{j} Q_{j}=\sum_{j \in \Omega} f_{j} Q_{j}+V^{\prime}
$$

где коэффициенты разложения имеют вид $f_{j}=\operatorname{tr}_{\mathrm{B}}\left(Q_{j}^{+} \rho_{\mathrm{B}}(T) V\right), \Omega$ обозначает полный набор частот, соответствующих системе, а оператор $V^{\prime}$ - это сумма всех слагаемых, содержащих прочие частоты. Данный оператор вносит нулевой вклад в динамику системы, поэтому в дальнейшем мы им пренебрегаем.

Теперь выразим коэффициенты разложения $f_{j}$ через операторы системы $F_{k}$, каждый из которых соответствует единственной частоте системы:

$$
\begin{gathered}
f_{j}=\sum_{k} C_{j k} F_{k}, \\
f_{j}(t)=\sum_{k} C_{j k} F_{k}(t)=\sum_{k} e^{-i \omega_{k} t} C_{j k} F_{k}, \quad F_{k}(t)=e^{-i \omega_{k} t} F_{k},
\end{gathered}
$$

где $C_{j k}-$ некоторые числа. 
Докажем что такое представление всегда существует. Пусть частота $\omega_{j}$ соответствует переходу между двумя собственными состояниями системы $|\mu\rangle$ и $|\nu\rangle$. Тогда, выбрав операторы $F_{k}$ и коэффициенты $C_{j k}$ в виде $F_{k}=|\mu\rangle\langle\nu|, C_{j k}=\left\langle\mu\left|f_{j}\right| \nu\right\rangle$, мы получим

$$
\begin{aligned}
\sum_{k} F_{k} C_{j k} & =\sum_{\mu} \sum_{\nu}|\mu\rangle\left\langle\nu\left|\left\langle\mu\left|f_{j}\right| \nu\right\rangle=\sum_{\mu} \sum_{\nu}\right| \mu\right\rangle\left\langle|\mu| f_{j} \mid \nu\right\rangle\langle\nu|= \\
& =\sum_{\mu}|\mu\rangle\left\langle\mu\left|f_{j} \sum_{\nu}\right| \nu\right\rangle\langle\nu|=f_{j} .
\end{aligned}
$$

С учетом (10) разложение (9) примет вид

$$
V=\sum_{k, \omega_{j} \in \Omega} C_{j k} F_{k} Q_{j}=\sum_{k} F_{k} \sum_{\omega_{j} \in \Omega} C_{j k} Q_{j},
$$

т.е. полученная факторизация оператора взаимодействия отражает разложение и по частотам системы (им соответствуют операторы $F_{j}$ ), и по частотам термостата (с ними связаны операторы $Q_{j}$ ).

Заметим, что во многих модельных задачах гамильтониан взаимодействия системы и термостата задается в виде (11) без всякого обоснования [10]-[12].

\section{4. МАТРИЦА КОРРЕЛЯЦИОННЫХ ФУНКЦИЙ}

Теперь рассмотрим уравнение $(4)$ в терминах операторов $F_{j}$ и $Q_{j}$. Раскрыв коммутаторы в подынтегральном выражении, получим в правой части этого уравнения четыре слагаемых:

$$
\begin{aligned}
\int_{0}^{\infty} & {\left[V_{\mathrm{I}}(t),\left[V_{\mathrm{I}}(t+\tau), \rho_{\mathrm{SI}}(t) \rho_{\mathrm{B}}(T)\right]\right] d \tau=\int_{0}^{\infty} V_{\mathrm{I}}(t) V_{\mathrm{I}}(t+\tau) \rho_{\mathrm{SI}}(t) \rho_{\mathrm{B}}(T) d \tau-} \\
& -\int_{0}^{\infty} V_{\mathrm{I}}(t) \rho_{\mathrm{SI}}(t) \rho_{\mathrm{B}}(T) V_{\mathrm{I}}(t+\tau) d \tau-\int_{0}^{\infty} V_{\mathrm{I}}(t+\tau) \rho_{\mathrm{SI}}(t) \rho_{\mathrm{B}}(T) V_{\mathrm{I}}(t) d \tau+ \\
& +\int_{0}^{\infty} \rho_{\mathrm{SI}}(t) \rho_{\mathrm{B}}(T) V_{\mathrm{I}}(t+\tau) V_{\mathrm{I}}(t) d \tau
\end{aligned}
$$

Второе слагаемое можно представить в виде

$$
\begin{aligned}
\int_{0}^{\infty} & \operatorname{tr}_{\mathrm{B}}\left[V_{\mathrm{I}}(t) \rho_{\mathrm{SI}}(t) \rho_{\mathrm{B}}(T) V_{\mathrm{I}}(t+\tau)\right] d \tau= \\
& =\sum_{j, k} F_{j}(t) \rho_{\mathrm{SI}}(t) \int_{0}^{\infty} F_{k}(t+\tau) \operatorname{tr}_{\mathrm{B}}\left(Q_{j} \rho_{\mathrm{B}}(T) Q_{k}(\tau)\right) d \tau= \\
& =\sum_{j, k} F_{j}(t) \rho_{\mathrm{SI}}(t) \int_{0}^{\infty} F_{k}(t+\tau) \mathbf{Q}_{j k}^{1}(t, \tau) d \tau
\end{aligned}
$$


или, воспользовавшись свойством (11), в виде

$$
\begin{aligned}
\int_{0}^{\infty} & \operatorname{tr}_{\mathrm{B}}\left(V_{\mathrm{I}}(t) \rho_{\mathrm{SI}}(t) \rho_{\mathrm{B}}(T) V_{\mathrm{I}}(t+\tau)\right) d \tau= \\
& =\sum_{j, k} F_{j}(t) \rho_{\mathrm{SI}}(t) F_{k}(t) \int_{0}^{\infty} e^{-i \omega_{k} \tau} \operatorname{tr}_{\mathrm{B}}\left(Q_{j} \rho_{\mathrm{B}}(T) Q_{k}(\tau)\right) d \tau= \\
& =\sum_{j, k} F_{j}(t) \rho_{\mathrm{SI}}(t) F_{k}(t) \mathbf{J}_{j k}\left(\omega_{k}, T\right) .
\end{aligned}
$$

Здесь функции $\mathbf{Q}_{j k}^{1}$ и $\mathbf{J}_{j k}$ - это элементы матрицы корреляционных функций соответственно во временно́м представлении,

$$
\mathbf{Q}_{j k}^{1}(\tau, T)=\operatorname{tr}_{\mathrm{B}}\left[Q_{j} \rho_{\mathrm{B}}(T) Q_{k}(\tau)\right]=\mathbf{Q}_{j k}(\tau, T),
$$

и в спектральном представлении,

$$
\mathbf{J}_{j k}(\omega, T)=\int_{0}^{\infty} \mathbf{Q}_{j k}(\tau, T) e^{-i \omega \tau} d \tau .
$$

Для остальных трех слагаемых в (12) корреляционные матрицы определяются аналогично:

$$
\begin{aligned}
& \mathbf{Q}_{j k}^{2}(\tau, T)=\operatorname{tr}_{\mathrm{B}}\left[Q_{k}(\tau) \rho_{\mathrm{B}}(T) Q_{j}\right]=\operatorname{tr}_{\mathrm{B}}\left[Q_{k} \rho_{\mathrm{B}}(T) Q_{j}(-\tau)\right]=\mathbf{Q}_{k j}(-\tau, T), \\
& \mathbf{Q}_{j k}^{3}(\tau, T)=\operatorname{tr}_{\mathrm{B}}\left[Q_{j} Q_{k}(\tau) \rho_{\mathrm{B}}(T)\right]=\operatorname{tr}_{\mathrm{B}}\left[Q_{k}(\tau) \rho_{\mathrm{B}}(T) Q_{j}\right]=\mathbf{Q}_{k j}(-\tau, T), \\
& \mathbf{Q}_{j k}^{4}(\tau, T)=\operatorname{tr}_{\mathrm{B}}\left[\rho_{\mathrm{B}}(T) Q_{k}(\tau) Q_{j}\right]=\operatorname{tr}_{\mathrm{B}}\left[Q_{j} \rho_{\mathrm{B}}(T) Q_{k}(\tau)\right]=\mathbf{Q}_{j k}(\tau, T) .
\end{aligned}
$$

Корреляционные матрицы (15) и (19) совпадают, а матрицы (17) и (18) получаются из них транспонированием и изменением знака времени.

Несмотря на то что спектр частот термостата, строго говоря, является дискретным, он в реальных случаях содержит довольно много значений и в силу этого может считаться квазинепрерывным. По этой причине спектральные функции можно описывать непрерывными функциями [13].

Заметим, что из уравнения (14) следует утверждение, высказанное в разделе 3: в разбиении (9) можно оставить только "резонансные" слагаемые. Так, в интеграле $\int_{0}^{\infty} e^{-i \omega_{k} \tau} \operatorname{tr}_{\mathrm{B}}\left(Q_{j} \rho_{\mathrm{B}}(T) Q_{k}(\tau)\right) d \tau$ "нерезонансные" слагаемые вносят нулевой вклад в $Q_{k}$, так как бесконечный верхний предел интегрирования вырезает все частоты, кроме $\omega_{k}$, после чего мы можем оставить и в $Q_{j}$ только резонансные слагаемые в силу ортогональности частотных операторов (см. комментарий к формуле (8)).

Здесь стоит подчеркнуть, что в точных уравнениях квантовой динамики следует писать переменный верхний предел $t$ интегрирования, который можно заменить на бесконечность при условии, что корреляционные функции имеют пренебрежимо малые значения на всей области определения, кроме некоторого узкого интервала $(0, \tau)[8]$. 
Для дальнейшего упрощения уравнения (12) рассмотрим свойства матрицы корреляционных функций $\mathbf{Q}_{j k}(\tau, T)=\operatorname{tr}_{\mathrm{B}}\left(Q_{j} \rho_{\mathrm{B}}(T) Q_{k}(\tau)\right)$ и ее фурье-образа $\mathbf{J}_{j k}(\omega, T)$ относительно операций транспонирования и обращения времени. Воспользовавшись формой (11) и ортонормированностью операторов $Q_{j}$, легко показать следующее:

1) обращение времени переводит $\mathbf{Q}$ в комплексно сопряженную матрицу и меняет знак частоты $\mathbf{J}$ :

$$
\mathbf{Q}_{j k}(-\tau, T)=\mathbf{Q}_{j k}^{*}(\tau, T), \quad \int_{0}^{\infty} Q_{j k}(-\tau, T) e^{-i \omega \tau} d \tau=J_{j k}(-\omega, T) ;
$$

2) транспонирование и обращение знака времени корреляционной функции приводит к появлению экспоненциального множителя в фурье-образе:

$$
\int_{0}^{\infty} Q_{k j}(-\tau, T) e^{-i \omega \tau} d \tau=J_{k j}(-\omega, T)=e^{-\hbar \omega / k T} J_{j k}(\omega, T) ;
$$

$3)$ при изменении операторов $Q_{j}$ на эрмитово сопряженные (т.е. при изменении знаков индексов на противоположные) фурье-образ корреляционной функции преобразуется так же, как при транспонировании: $J_{-j,-k}(\omega, T)=e^{\hbar \omega / k T} J_{j k}(-\omega, T)$, $J_{k j}(\omega, T)=J_{-j,-k}(-\omega, T)$.

С учетом этих свойств функций $\mathbf{Q}$ и $\mathbf{J}$ уравнение (12) примет вид

$$
\begin{aligned}
\int_{0}^{\infty} \operatorname{tr}_{\mathrm{B}}\left[V_{\mathrm{I}}(t),\left[V_{\mathrm{I}}(t+\tau), \rho_{\mathrm{SI}}(t) \rho_{\mathrm{B}}(T)\right]\right] d \tau= \\
=\sum_{j, k} J_{-j,-k}\left(-\omega_{k}, T\right) F_{j}(t) F_{k}(t) \rho_{\mathrm{SI}}(t)-\sum_{j, k} F_{j}(t) \rho_{\mathrm{SI}}(t) F_{k}(t) J_{j k}\left(\omega_{k}, T\right)- \\
\quad-\sum_{j, k} J_{-j,-k}\left(-\omega_{k}, T\right) F_{k}(t) \rho_{\mathrm{SI}}(t) F_{j}(t)+\sum_{j, k} \rho_{\mathrm{SI}}(t) J_{j k}\left(\omega_{k}, T\right) F_{k}(t) F_{j}(t) .
\end{aligned}
$$

Теперь с помощью этого уравнения мы можем перейти от матриц, содержащих функции частот или времени, к одной числовой матрице:

$$
\mathbf{J}_{j k}(T)=\mathrm{J}_{j k}\left(\omega_{k}, T\right), \quad \mathbf{J}_{-j,-k}(T)=\mathrm{J}_{-j,-k}\left(-\omega_{k}, T\right) .
$$

Тогда выражение для диссипативного члена в уравнении Редфилда примет вид

$$
\begin{aligned}
\int_{0}^{\infty} & \operatorname{tr}_{\mathrm{B}}\left[V_{\mathrm{I}}(t),\left[V_{\mathrm{I}}(t+\tau), \rho_{\mathrm{SI}}(t) \rho_{\mathrm{B}}(T)\right]\right] d \tau= \\
= & \sum_{j, k} J_{-j,-k}(T) F_{j}(t) F_{k}(t) \rho_{\mathrm{SI}}(t)-\sum_{j, k} J_{j, k}(T) F_{j}(t) \rho_{\mathrm{SI}}(t) F_{k}(t)- \\
& \quad-\sum_{j, k} J_{-j,-k}(T) F_{k}(t) \rho_{\mathrm{SI}}(t) F_{j}(t)+\sum_{j, k} \rho_{\mathrm{SI}}(t) J_{j, k}(T) F_{k}(t) F_{j}(t) ;
\end{aligned}
$$

переходя к сопряженным операторам, мы можем переписать его как

$$
\begin{aligned}
& \int_{0}^{\infty} \operatorname{tr}_{\mathrm{B}}\left[V_{\mathrm{I}}(t),\left[V_{\mathrm{I}}(t+\tau), \rho_{\mathrm{SI}}(t) \rho_{\mathrm{B}}(T)\right]\right] d \tau= \\
&=\sum_{j, k} J_{j, k}(T) F_{j}^{+}(t) F_{k}^{+}(t) \rho_{\mathrm{SI}}(t)-\sum_{j, k} J_{j, k}(T) F_{j}(t) \rho_{\mathrm{SI}}(t) F_{k}(t)- \\
& \quad-\sum_{j, k} J_{j, k}(T) F_{k}^{+}(t) \rho_{\mathrm{SI}}(t) F_{j}^{+}(t)+\sum_{j, k} \rho_{\mathrm{SI}}(t) J_{j, k}(T) F_{k}(t) F_{j}(t) .
\end{aligned}
$$


Это одно из основных уравнений в настоящей статье. Из него видно, что вся информация о влиянии термостата на систему может быть записана в единственной матрице $\mathbf{J}(T)$ (23). Эта матрица во многом аналогична матрицам $\Gamma_{\mu \nu \lambda \kappa}^{+}$и $\Gamma_{\mu \nu \lambda \kappa}^{-}$, используемым при поэлементном (численном) решении уравнения Редфилда. Однако стоит заметить, что, во-первых, размерность $\mathbf{J}(T)$ может быть ниже (число частот меньше или равно числу пар элементов), а во-вторых, используется только одна матрица вместо двух.

\section{5. ДИАГОНАЛИЗАЦИЯ КОРРЕЛЯЦИОННОЙ МАТРИЦЫ}

Для уменьшения числа слагаемых в уравнении (25) проведем диагонализацию матрицы J. Поскольку она не является эрмитовой, у нее существуют два различных набора собственных векторов с одними и теми же собственными значениями правый и левый:

$$
\mathbf{J}(T) \bar{X}_{n}(T)=J_{n}(T) \bar{X}_{n}(T), \quad \bar{Y}_{n}(T) \mathbf{J}(T)=J_{n}(T) \bar{Y}_{n}(T) .
$$

Наложим на них следующее условие нормировки: $\mathbf{Y}^{+}(T) \mathbf{X}(T)=\mathbf{1}$, тогда

$$
\mathbf{Y}^{+}(T) \mathbf{J}(T) \mathbf{X}(T)=\left(\begin{array}{cccc}
J_{1}(T) & 0 & \ldots & 0 \\
0 & J_{2}(T) & \ldots & 0 \\
\vdots & \vdots & \ddots & \vdots \\
0 & 0 & \ldots & J_{n}(T)
\end{array}\right)=\mathbf{J}_{\mathrm{d}}(T)
$$

где индекс "d" обозначает диагональную форму, или

$$
\mathbf{J}(T)=\mathbf{X}(T) \mathbf{J}_{\mathrm{d}}(T) \mathbf{Y}^{+}(T)
$$

Используя это представление, преобразуем уравнение (25). Операторы системы $F_{n}$ представим в векторном виде

$$
\bar{F}=\left(\begin{array}{c}
F_{-n} \\
\vdots \\
F_{n}
\end{array}\right), \quad \bar{F}^{+}=\left(\begin{array}{c}
F_{-n}^{+} \\
\vdots \\
F_{n}^{+}
\end{array}\right) \text {, }
$$

тогда уравнение (25) примет форму

$$
\begin{aligned}
\int_{0}^{\infty} \operatorname{tr}_{\mathrm{B}}\left[V_{\mathrm{I}},\left[V_{\mathrm{I}}(\tau), \rho_{\mathrm{SI}}(t) \rho_{\mathrm{B}}(T)\right]\right] d \tau=\bar{F}^{+} \mathbf{J}(T) \bar{F}^{+} \rho_{\mathrm{SI}}(t)-\bar{F} \mathbf{J}(T) \rho_{\mathrm{SI}}(t) \bar{F}- \\
\quad-\bar{F}^{+} \mathbf{J}^{+}(T) \rho_{\mathrm{SI}}(t) \bar{F}^{+}+\rho_{\mathrm{SI}}(t) \bar{F} \mathbf{J}^{+}(T) \bar{F} .
\end{aligned}
$$


Теперь, воспользовавшись соотношением (26) и его эрмитово сопряженной формой, получим

$$
\begin{aligned}
\bar{F} \mathbf{J}^{+}(T) \bar{F} & =\bar{F} \mathbf{X}(T) \mathbf{J}_{\mathrm{d}}(T) \mathbf{Y}^{+}(T) \bar{F}= \\
& =\left(\ldots \sum_{j} X_{j \alpha}(T) F_{j} \ldots\right) \mathbf{J}_{\mathrm{d}}(T)\left(\begin{array}{c}
\vdots \\
\sum_{j} Y_{j \alpha}(T) F_{j} \\
\vdots
\end{array}\right) \\
\bar{F}^{+} \mathbf{J}^{+}(T) \bar{F}^{+} & =\bar{F}^{+} \mathbf{Y}(T) \mathbf{J}_{\mathrm{d}}(T) \mathbf{X}^{+}(T) \bar{F}^{+}= \\
& =\left(\ldots \sum_{j} Y_{j \alpha}(T) F_{j}^{+} \ldots\right) \mathbf{J}_{\mathrm{d}}(T)\left(\begin{array}{c}
\sum_{j} X_{j \alpha}(T) F_{j}^{+} \\
\vdots
\end{array}\right),
\end{aligned}
$$

где векторы, стоящие в правой части каждого уравнения, получаются из $\bar{F}$ и $\bar{F}^{+}$ действием на них матриц $X$ и $Y$. Элементы этих векторов будем называть коррелированными операторами диссипации и обозначим $X_{\alpha}$ и $Y_{\alpha}$ :

$$
X_{\alpha}=\sum_{j} X_{j \alpha}(T) F_{j}, \quad Y_{\alpha}=\sum_{j} Y_{j \alpha}(T) F_{j} .
$$

Тогда уравнение (27) запишется как

$$
\begin{aligned}
\int_{0}^{\infty} & \operatorname{tr}_{\mathrm{B}}\left[V_{\mathrm{I}},\left[V_{\mathrm{I}}(\tau), \rho_{\mathrm{SI}}(t) \rho_{\mathrm{B}}(T)\right]\right] d \tau=\sum_{\alpha} J_{\alpha} X_{\alpha}^{+} Y_{\alpha}^{+} \rho_{\mathrm{SI}}(t)-\sum_{\alpha} J_{\alpha} X_{\alpha} \rho_{\mathrm{SI}}(t) Y_{\alpha}- \\
& -\sum_{\alpha} J_{\alpha} Y_{\alpha}^{+} \rho_{\mathrm{SI}}(t) X_{\alpha}^{+}+\sum_{\alpha} \rho_{\mathrm{SI}}(t) J_{\alpha} Y_{\alpha} X_{\alpha} .
\end{aligned}
$$

С учетом этого соотношения уравнение Редфилда для редуцированной матрицы плотности (4) примет вид

$$
\begin{aligned}
\frac{d}{d t} \rho_{\mathrm{SI}}(t)= & -\frac{1}{\hbar^{2}} \sum_{\alpha} J_{\alpha} X_{\alpha}^{+}(t) Y_{\alpha}^{+}(t) \rho_{\mathrm{SI}}(t)+\frac{1}{\hbar^{2}} \sum_{\alpha} J_{\alpha} X_{\alpha}(t) \rho_{\mathrm{SI}}(t) Y_{\alpha}(t)+ \\
& +\frac{1}{\hbar^{2}} \sum_{\alpha} J_{\alpha} Y_{\alpha}^{+}(t) \rho_{\mathrm{SI}}(t) X_{\alpha}^{+}(t)-\frac{1}{\hbar^{2}} \sum_{\alpha} J_{\alpha} \rho_{\mathrm{SI}}(t) Y_{\alpha}(t) X_{\alpha}(t)
\end{aligned}
$$

в представлении взаимодействия и вид

$$
\begin{aligned}
\frac{d}{d t} \rho_{\mathrm{S}}(t)= & -\frac{i}{\hbar}\left[H_{\mathrm{S}}, \rho_{\mathrm{S}}(t)\right]-\frac{1}{\hbar^{2}} \sum_{\alpha} J_{\alpha} X_{\alpha}^{+} Y_{\alpha}^{+} \rho_{\mathrm{S}}(t)+\frac{1}{\hbar^{2}} \sum_{\alpha} J_{\alpha} X_{\alpha} \rho_{\mathrm{S}}(t) Y_{\alpha}+ \\
& +\frac{1}{\hbar^{2}} \sum_{\alpha} J_{\alpha} Y_{\alpha}^{+} \rho_{\mathrm{S}}(t) X_{\alpha}^{+}(t)-\frac{1}{\hbar^{2}} \sum_{\alpha} J_{\alpha} \rho_{\mathrm{S}}(t) Y_{\alpha} X_{\alpha}
\end{aligned}
$$

в представлении Шредингера. Соотношения (30) и (31) и есть новая форма уравнения Редфилда для диссипативной динамики. В ней диссипативный член выражен 
через операторы, соответствующие собственным векторам матрицы корреляционных функций. В следующей статье на примере простой модели мы покажем, что эта форма записи уравнения Редфилда обладает определенными преимуществами перед традиционной.

Важно отметить, что уравнение (31) можно использовать для определения операторов $L_{j}$ в уравнении Линдблада [14]

$$
\begin{aligned}
\frac{d}{d t} \rho_{\mathrm{S}}(t)= & -\frac{i}{\hbar^{2}}\left[H_{\mathrm{S}}, \rho_{\mathrm{S}}(t)\right]-\frac{1}{\hbar^{2}} \sum_{\alpha} L_{j}^{+} L_{j} \rho_{\mathrm{S}}(t)+\frac{1}{\hbar^{2}} \sum_{\alpha} L_{j} \rho_{\mathrm{S}}(t) L_{j}^{+}+ \\
& +\frac{1}{\hbar^{2}} \sum_{\alpha} L_{j} \rho_{\mathrm{S}}(t) L_{j}^{+}-\frac{1}{\hbar^{2}} \sum_{\alpha} \rho_{\mathrm{S}}(t) L_{j}^{+} L_{j},
\end{aligned}
$$

т.е. операторы $\mathbf{X}$ и $\mathbf{Y}$ можно представить как линейную комбинацию операторов $\mathbf{L}$. В случае когда операторы $\mathbf{X}$ и $\mathbf{Y}$ эрмитово сопряжены друг другу, они являются операторами Линдблада: $X_{\alpha}=Y_{\alpha}^{+}=L_{\alpha} / \sqrt{J_{\alpha}}$.

\section{6. АНАЛИЗ МНИМОЙ ПОПРАВКИ К ГАМИЛЬТОНИАНУ}

Для анализа уравнения (31) мы можем перегруппировать слагаемые следующим образом:

$$
\begin{aligned}
\frac{d}{d t} \rho_{\mathrm{S}}(t)=- & \frac{i}{\hbar}\left[\left(H_{\mathrm{S}}-\frac{i}{\hbar} \sum_{\alpha} J_{\alpha} X_{\alpha}^{+} Y_{\alpha}^{+}\right) \rho_{\mathrm{S}}(t)\right]+\frac{i}{\hbar}\left[\rho_{\mathrm{S}}(t)\left(H_{\mathrm{S}}+\frac{i}{\hbar} \sum_{\alpha} J_{\alpha} Y_{\alpha} X_{\alpha}\right)\right]+ \\
& +\frac{1}{\hbar^{2}} \sum_{\alpha} J_{\alpha} X_{\alpha} \rho_{\mathrm{S}}(t) Y_{\alpha}+\frac{1}{\hbar^{2}} \sum_{\alpha} J_{\alpha} Y_{\alpha}^{+} \rho_{\mathrm{S}}(t) X_{\alpha}^{+}
\end{aligned}
$$

и ввести комплексную поправку к гамильтониану системы:

$$
H(T)=H_{\mathrm{S}}-\frac{i}{\hbar} \sum_{\alpha} J_{\alpha} X_{\alpha}^{+} Y_{\alpha}^{+} .
$$

Тогда уравнение Редфилда для редуцированной матрицы плотности примет вид

$$
\begin{aligned}
\frac{d}{d t} \rho_{\mathrm{S}}(t)=- & \frac{i}{\hbar}\left[H(T) \rho_{\mathrm{S}}(t)\right]+\frac{i}{\hbar}\left[\rho_{\mathrm{S}}(t) H^{+}(T)\right]+ \\
& +\frac{i}{\hbar^{2}} \sum_{\alpha} J_{\alpha} X_{\alpha} \rho_{\mathrm{S}}(t) Y_{\alpha}+\frac{i}{\hbar^{2}} \sum_{\alpha} J_{\alpha} Y_{\alpha}^{+} \rho_{\mathrm{S}}(t) X_{\alpha}^{+} .
\end{aligned}
$$

Новый оператор $H(T)$ имеет смысл оператора комплексной энергии, или эффективного оператора Гамильтона с мнимой поправкой, введенной для учета диссипации. Эта поправка также может быть определена на основании только формы энергии взаимодействия:

$$
H(T)-H_{\mathrm{S}}=-\frac{i}{\hbar} \int_{0}^{\infty} \operatorname{tr}_{\mathrm{B}}\left(V V_{\mathrm{I}}(\tau) \rho_{\mathrm{B}}(T)\right) d \tau .
$$

Эрмитовость оператора $\sum_{\alpha} J_{\alpha} X_{\alpha}^{+} Y_{\alpha}^{+}$можно показать, используя теорему Линдблада. 
Важно заметить, что в рамках теории Редфилда при введении мнимых поправок в гамильтониан в уравнении динамики появляется дополнительное слагаемое

$$
\frac{1}{\hbar^{2}} \sum_{\alpha} J_{\alpha} X_{\alpha} \rho_{\mathrm{S}}(t) Y_{\alpha}+\frac{1}{\hbar^{2}} \sum_{\alpha} J_{\alpha} Y_{\alpha}^{+} \rho_{\mathrm{S}}(t) X_{\alpha}^{+},
$$

которое необходимо для сохранения нормировки матрицы плотности. Это слагаемое также показывает, на какие именно колебательные уровни перераспределяется заселенность, убывающая из-за появления мнимых поправок к энергии. Следовательно, операторы $\mathbf{X}$ и $\mathbf{Y}$ имеют смысл операторов переходов, формирующих диссипативную часть гамильтониана:

$$
H(T)-H_{\mathrm{S}}=-\frac{i}{\hbar} \sum_{\alpha} J_{\alpha} X_{\alpha}^{+} Y_{\alpha}^{+} .
$$

Также стоит отметить, что существует бесконечное число линейных преобразований операторов $\mathbf{X}$ и $\mathbf{Y}$, не меняющих вида уравнения (31). Однако, только операторы (28), полученные диагонализацией матрицы корреляционных функций, имеют смысл коррелированных операторов диссипации, т.е. соответствуют собственным векторам матрицы $\mathbf{J}(T)(23)$.

\section{7. ЗАКЛЮЧЕНИЕ}

Предложена новая форма уравнения Редфилда, основанная на построении и диагонализации матрицы корреляционных функций. Показано, что на основании разложения (5) оператора взаимодействия системы с термостатом на слагаемые, соответствующие небольшому числу переходов внутри системы, можно существенно сократить размерность задачи о диссипативной динамике. Этот подход может быть применен к ряду задач, при решении которых можно исходить из простых приближений (гармоническое приближение для описания колебательных состояний системы, билинейная форма взаимодействия система-термостат и т.п.) и аналитически решаемых задач [15]. Также стоит заметить, что при моделировании динамики макромолекулярных систем всегда можно произвести разбиение на подсистемы, для которых допустимо применение простых приближений.

Подробно рассмотрена процедура построения и диагонализации матрицы корреляционных функций. Получена новая форма (30), (31) уравнения Редфилда, которая упрощает рассмотрение задачи о диссипативной динамике в случае, когда возможен выбор основных частот перехода. Пример применения этой процедуры будет рассмотрен в будущей статье.

В заключительной части статьи проведен анализ уравнения (31) на основании выделения мнимой поправки к гамильтониану. Полученное уравнение (34) может быть также полезно при феноменологическом введении мнимых поправок к энергиям, поскольку показана необходимость введения дополнительных компенсирующих слагаемых. 
Благодарности. Работа выполнена при частичной поддержке РФФИ (грант № 06-03-32921).

\section{Список литературы}

[1] V. Sundström, Progr. Quantum Electron., 24:5 (2000), 187-238.

[2] R.S. Knox, J. Photochem. Photobiol. B, 49:2-3 (1999), 81-88.

[3] C. E. Crespo-Hernández, B. Cohen, P. M. Hare, B. Kohler, Chem. Rev., 104:4 (2004), 1977-2020.

[4] S. K. Pal, A. H. Zewail, Chem. Rev., 104:4 (2004), 2099-2124.

[5] X. Hu, T. Ritz, A. Damjanovic, K. Schulten, J. Phys. Chem. B, 101:19 (1997), 3854-3871.

[6] W. Domcke, G. Stock, "Theory of ultrafast nonadiabatic excited-state processes and their spectroscopic detection in real time", Advances in Chemical Physics, 100, eds. I. Prigogine, S. A. Rice, Wiley, New York, 1997, 1-169.

[7] A. G. Redfield, Adv. Magn. Res., 1 (1965), 1-32.

[8] К. Блум, Теория матриць плотности и ее приложения, Мир, М., 1983.

[9] W. H. Louisell, Quantum Statistical Properties of Radiation, Reprint of the 1973 ed., Wiley Classics Lib., Wiley, New York, 1990.

[10] D. Egorova, A. Kühl, W. Domcke, Chem. Phys., 268:1-3 (2001), 105-120.

[11] D. Egorova, W. Domcke, J. Photochem. Photobiol. A, 166:1-3 (2004), 19-31.

[12] A. Kühl, W. Domcke, J. Chem. Phys., 116:1 (2002), 263-274.

[13] U. Weiss, Quantum Dissipative Systems, 2nd ed., Ser. Modern Condensed Matter Phys., 10, World Sci., Singapore, 1999.

[14] G. Lindblad, Comm. Math. Phys., 48:2 (1976), 119-130.

[15] В. В. Еремин, И. О. Глебов, ТМФ, 153:1 (2007), 130-144.

Поступила в редакцию 29.10.2008, после доработки 10.12.2008 